\title{
Efficient Vector-oriented Graphic Drawing Method For Laser-scanned Display
}

\author{
O. Halabi and N. Chiba \\ Department of Computer and Information Sciences, Iwate University, Japan
}

\begin{abstract}
Laser projection, such as that used in laser light shows, usually uses computer-drawn graphics.

However, some restrictions are imposed by laser-scanned displays on these vector-based drawings.

This paper presents a general vector-oriented drawing method to display computer vector graphics on different laser-scanned displays with optimization. The method considers how to solve the three fundamental problems inherent in laser projection: achieving constant beam intensity, blanking timing and corner overshooting. A velocity parameter has been used to achieve constant beam velocity for all strokes. Blanking timing which is related to turning on/off the laser beam has been examined and a simple test pattern is presented to estimate the total delay that exists in the laserscanned display and compensate for it. In addition, an optimized approach to avoid corner overshooting and ringing is proposed: the dwelling time of the laser beam at a corner is set according to the corner angle. Moreover, a comparison between the proposed method and the point-oriented method in terms of speed has been presented. It is demonstrated that the vector-oriented approach outperforms the point-oriented approach. Thus, this paper describes the fundamental problems related to laser graphics that must be well considered when drawing with any laser-scanned display.
\end{abstract}

Keywords: Laser Graphics, Vector Graphics, Drawing Optimization, Methodology and TechniquesStandards.

\section{Introduction}

Laser-scanned displays (LSD) have unique and significant advantages that make them a viable and desirable medium. They can be used in many research fields in order to develop new approaches for conveying 
information and graphics on almost any surface and at any distance. Despite their limitations, LSDs have recently been attracting much attention due to the advancement made in laser technology and electronics that make it possible to manufacture ever smaller projectors with faster speed. Therefore, the method by which a laser display generates graphics from vector data needs to be optimized by taking into account the uniqueness and inherent problems of such displays to achieve the best performance.

Many applications can employ and benefit from this technology. In entertainment, a recent example is the show organized in Siggraph 2007 where a state-of-the-art laser projection system designed to show real-time vector graphics was used for the first time to play several classic arcade games on a giant projection screen [1]. In Medical, Glossop et al. [2] have used a laser projector to display information directly on a patient as a 'projection screen'. In industry, an LSD was specifically designed to transfer the pictures, figures and graphics of desired variety, size and intensity on all types of surfaces in textile manufacturing at desired precision [3]. LSDs are also used in industrial application where accuracy is an important issue. Applications of such type of laser projection could be component placement, structural assembly, edge trimming and viewing invisible substructure or hidden components in many manufacturing processes [4]. In display's technology, Pryor et al. developed a new display technology called the virtual retinal display (VRD) that scans modulated low-energy laser light directly onto the viewer's retina to create a perception of a virtual image [5] [6]. Edgar el al. [7] studied the effect of viewing an LSD on color perception, and Kelly et al. [8] provided a comparison between an LSD and CRT in terms of flicker sensitivity. The development of a laser-scanning technique to produce 3D images floating in air, or what is commonly known as a hologram, has also gained momentum. A 3D laser display based on a liquid-crystal medium was proposed in [9], and AIST used the plasma produced in air and a laser-scanning system to shoot the plasma and produce real 3D images in the air [10]. Recently, in our laboratory, we have conducted a primarily research into laser graphics, hoping to attract many researchers into the field. Menendez et al. presented a vector-based method to display Bezier curves on an LSD [11]. Abderyim et al. proposed a morphing technique to simulate candle animation for an LSD [12].

\subsection{Related works}


Many research activities are focused on employing LSDs for different applications; however, to the best of the authors' knowledge, no academic research exists on the fundamental aspects and the drawing algorithms that specifically address the laser display or laser graphics. It may be argued that many studies were conducted in the field of vector graphics and a vector display devices such as a plotter that is almost obsolete [13][14][15][16]. However, a laser display has unique features such as blanking and producing different curvature according to the different scanning speeds at the corners and endpoints of an image. These features make the previous research about vector displays inapplicable to laser displays. Further, the significant advances in computer graphics can be translated to laser graphics to create new capabilities and features.

Much of the information available on the Web is related mostly to laser show business and commercial software. Since laser graphics is mostly used in entertainment, most of the available software is commercial and intended for programming laser shows [17][18][19][20]. We believe that it is possible to optimize and add a new functionality using 3D graphics technology to fully utilize laser displays. In one paper, a laser display was seriously discussed, and it is worth mentioning here that the paper is written by the founders of Pangolin laser show software [21]. The authors discussed the advantages of their laser display and the difference between laser graphics and raster graphics. Further, they mentioned their approach to draw graphics using point-oriented method that is used in their Pangolin software [21]. The most common approach that can be found in almost all commercial laser software is a point-oriented approach in which the software creates blanked, anchor and midstroke points to help achieve constant scan velocity. The previous method required that many extra points be added at the corners of images and also when turning the laser on and off. In addition, it was necessary to interpolate a stroke into uniformly divided small strokes. However, a question that might be raised here that whether such an approach is appropriate since the addition of many points limits the already small bandwidth of the galvanometer scanners. There is no such evaluation or comparison data available, even though it is considered as the de facto standard in the industry.

\subsection{Methods}

This paper presents a general vector-oriented method to draw vector computer graphics on different LSDs with optimization. As opposed to point-oriented approach, this method considers drawing the strokes directly from 
the vector data without the need to add any additional mid-stroke points. In order to achieve a perfect laser projection method, we need to consider how to tackle three fundamental problems inherent with laser projection: The first problem is achieving constant beam intensity for all strokes; otherwise, a short stroke will look brighter than a long stroke. We propose a novel approach in which the velocity of a laser beam is controlled according to the length of each stroke. A velocity parameter which describes the motion speed of the laser beam has been used to control the laser drawing software to assign a proper time to each stroke. This approach waives the need to add extra mid-stroke points to achieve constant beam intensity as done by the point-oreinted method. The second problem is about the blanking timing, which is related to turning on and off the beam. The timing of blanking at each endpoint can significantly affect the quality of the projected graphics and produce undesired effects. This is because of the difference between the speed of the intensity control devices and X-Y scanners. Few systems have hardware compensation for this time difference; therefore, the required timing adjustment must be done via software. However, systems designed to work with any projector must have a technique to compensate for this time lag for different laser projectors. We propose a simple test that would enable us to accurately set the most suitable time for drawing each vector and the compensation time for blanking. The third problem is the need to compensate laser graphics at the corners and endpoints in order to avoid overshooting and ringing. The laser beam must dwell at corners;in the point-oriented approach, the dwell time is achieved by adding extra points at the corner. The number of these points are the same, regardless of the angle between the lines that define the corner. In this work, we popose an optimaization approach to set the dwelling time according to the corner angle. In this way we could optimize the dwelling time at the corners more efficiently.

This paper extensively addresses the three above problems and present novel solutions for the first two problems and an optimization approach for the third problem. The three new approaches are used to achieve a perfect and efficient vector-oriented laser projection method. An evaluation of each method is discussed, and an overall performance of the proposed drawing method compared to the point-oriented method is deeply explored.

\section{Experimental laser projector}

A laser display is an essential part of a complete computer laser graphics system. A laser display was built in our Laboratory (Figure 1), which consists of the following components: 


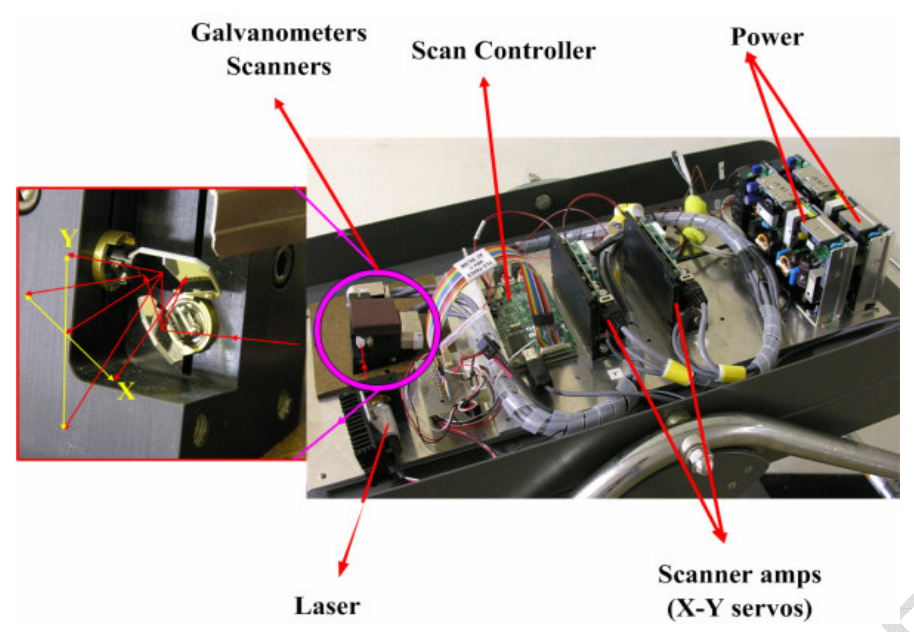

Figure 1: Experimental Laser projector.

Scan controller: It is responsible for communicating with PC and axis servos, and it has memory to store programs before sending them to the scanner amp.

Scanner amp: It converts the digital signals received from the main controller into analogue currents for the galvanometers and also communicates the position feedback to the scan controller. Two scanner amps are required to run $\mathrm{X}-\mathrm{Y}$ scanners.

Galvanometer scanner: It consists of a tiny mirror that deflects the laser beam. Two scanners are required, one for the horizontal motion $(\mathrm{X})$ and one for the vertical motion $(\mathrm{Y})$. Together, they can position the beam anywhere on the display screen.

Laser beam: A laser beam of a given colour and intensity.

Power: Supply power to the laser projector.

The projector used in our experiments was constructed using the following components [22][23]:

Scan controller: GSI Lumonics SC2000.

Servo controllers (scan amps): GSI Lumonics MiniSAX.

Galvanometers scanners: GSI Lumonics VM-500.

Laser: Coherent MVP control diode-laser module. Wavelength: $670 \mathrm{~nm}$ (red). Power: $0.95 \mathrm{~mW}$. 


\section{Considerations for laser graphics drawings}

\subsection{Constant Intensity}

An inherent problem in laser projection is achieving a constant velocity of the laser. This is related to the strong relation between the drawing speed of each line segment (stroke) and the resulting projected-beam intensity. If two lines of different lengths are drawn with the same time interval, the shorter line will look brighter than the longer one because to draw the longer line in the same time interval, the mirrors must be moved faster than when drawing the shorter line. The slower the mirrors move, the brighter (more intense) the laser beam will look. In principle, this problem can be solved by either moving the laser beam with a constant velocity for all vectors or by applying constant laser beam intensity and adjusting the velocity according to the distance the laser has to travel from one point to another.

\subsection{Blanking timing}

Blanking is the technique of turning a laser beam on and off with precise control. For scanned graphics, blanking allows images to have disconnected sections where the beam is hidden. Blanking can be digital (on/off) or analogue (continuous intensity control). Blanking can be performed using a dedicated scanner, an acoustooptic modulator (AOM), or by electronically controlling the laser output as done with semiconductor lasers. Blanking timing is a critical issue for an LSD. This is mainly because of the difference between the speeds of intensity devices and the associated X-Y scanner: the former operate faster than the latter. This implies that a command to turn the beam off at a certain point will take effect before the beam reaches that location. This time lag should be considered carefully in order to achieve correct projection with blanking. The most significant delays occur between the time when an instruction is evaluated and when an actual voltage value emerges from the output of a DAC (digital-to-analogue converter). There will be further critical delay that is difficult to be easily estimated between the instant the command is received by the X-Y scanners and the instant a mirror has actually moved into the desired position. For achieving correct command timing, each projection system needs to be evaluated to estimate and compensate for these lag/delay times. We call all these delays the laser display latency. An experimental demonstration of how to estimate and compensate this latency will be presented in the blanking experiment section. 


\section{Point-oriented method}

Laser vectors cannot be stroked as fast as CRT vectors. For example, in laser graphics, a laser beam must dwell at corners and at stroke end points for about $300 \mathrm{~ms}$ in order to avoid overshooting and ringing. To the best of our knowledge, with the lack of papers describing how the industry is tackling this problem, we can say that there are two approaches: (1) Using software, extra points are added to an image in order to achieve constant velocity. (2) For stroke generation, dedicated hardware is used instead of software.

A typical line might have 22 points: at the line start location, there will be 5 blanked points that are followed by 3 visible points ( called 'anchor' points); the line itself is interpolated to many points that lie along the line and has other 3 visible and 5 blanked points at the end[21]. This method simply depends on adding a large number of points to compensate the velocity.

\subsection{Scan rate}

One of the most frequently used terms in the discussion of scanner performance is ' $30 \mathrm{~K}$ pps', which denotes 30,000 points per second. Laser computer systems generate laser graphics by outputting XY data points sequentially. These data are used to move the scanners along the horizontal (X) and vertical (Y) axes to draw laser graphics. The rate at which these data points are output is defined as 'points per second' or 'pps'; therefore, $30 \mathrm{~K}$ pps indicates an output rate of 30,000 points per second from the computer. Other terms used to describe the rate at which the points are output include 'scanning speed', 'scan rate' and 'point rate'. This parameter is specifically related to LSDs and must be considered carefully when creating graphics by the point-oriented approach for generating flicker-free drawings. PPS can be calculated from Equation (1):

$$
P P S=N O P \times F P S
$$

$P P S$ is the scan rate in points per seconds; NOP, the number of points in one frame drawing; and FPS, the update rate in frames per second for each frame.

To draw in this approach we first need to have the whole data for the frame to be drawn before we can apply the Equation (2): 


$$
t=\frac{d}{D \times F P S}
$$

where $t$ is the drawing time delay in time units for a given Vector; $d$, the length of a the vector in distance units; $D$, the sum of all the vector's length for a one frame; and FPS, the desired update rate in frames per second. The draw back of this approach is the need to process the whole vetor data of the frame to be drawn before you can assign the suitable time for each stroke; therefore, it is not suitable for real time processing and analyzing.

\section{Vector-Oriented method}

As opposed to the point-oriented approach, this method considers drawing the strokes directly from vector data without the need to add any additional points. In order to achieve perfect laser-scanned projection, every drawing method needs to consider how to tackle three fundamental problems that are inherent in laser graphics: First, achieving constant beam intensity for all strokes; second, the need to compensate the laser graphics at the corners and the endpoints in order to avoid overshooting and ringing; and third, achieving accurate blanking timing.

\subsection{Velocity Concept}

As mentioned in earlier, achieving a constant projected-beam intensity of the laser beam is an inherent problem in laser projection. A velocity parameter that describes the motion speed of a laser beam has been used to achieve constant beam intensity by assigning a proper drawing time for each stroke according to its length. In this approach, there is no need to wait until you process all the data, you can calculate the time for each stroke on the fly using the velocity Equation (3):

$$
t=\frac{d}{V}
$$

where $t$ is the time assigned to the beam to move from the start point to the end point of each vector; $d$, the vector length; and $V$, the beam velocity. Compared to the way of calculating $t$ in Equation (2), it is clear that there is no need to calculate $D$, the total length of one frame vectors. This makes the proposed approach suitable for real time application where there is no need to wait for one frame data to be processed until you can draw on 
LSD. Moreover, by changing $V$, we can draw graphics at different speeds with constant beam velocity. Figure 2 illustrate the two projected images: (a) before applying velocity approach and (b) after applying velocity approach. In this approach, the update rate (FPS) for each frame depends on the suggested velocity parameter as opposed to PPS in Equation (1); therefore, velocity can be considered to describe the scanning speed in vectororiented approach as opposed to the scan rate in point-oriented approach.

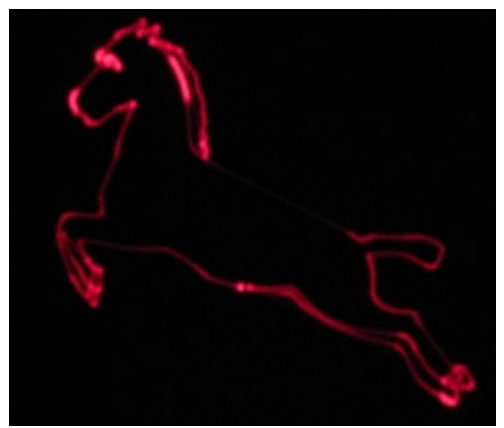

(a)

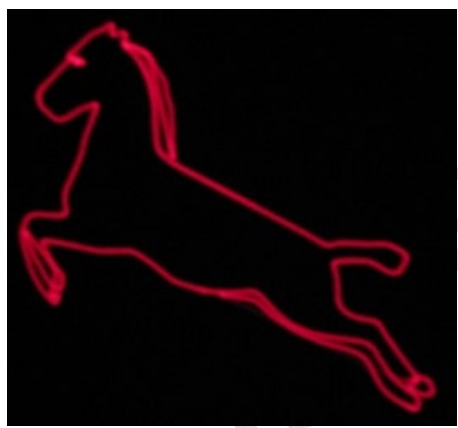

(b)

Figure 2: Before and after applying velocity concept.

\subsubsection{Efficiency}

The main advantage of this approach is waiving the need to insert extra mid-points, which results in decreasing the number of points in each drawing frame dramatically. To show the performance of this approach, four drawings produced by commercial software in point-mode has been converted to vector-based drawing, where extra mid-points, extra end points, and extra points at the corners has been removed. Figure 3 shows the drawings used to calculate the efficiency. As can be seen in Table 1, performance differs from image to image. Drawings with more straight lines (e.g. sun flower) have better performance than drawings with more curved lines (e.g. goofy), $68.8 \%$ to $24.5 \%$, respectively. Never the less, the average of deleted points is $52.8 \%$, which proves the significance of the proposed approach. 


\section{ACCEPTED MANUSCRIPT}

10

Table 1: The efficiency of velocity approach in decreasing the number of points in each drawing frame.

\begin{tabular}{|c|c|c|c|c|c|}
\hline & \multicolumn{4}{|c|}{ NOP (Number of Points) } & \multirow{2}{*}{ Average } \\
\hline Image Name & Castle & Flower & Sun Flower & Goofy & \\
\hline Point-based drawing & 1612 & 1032 & 1315 & 1073 & 1258 \\
\hline Vector-based drawing & 578 & 566 & 410 & 810 & 593 \\
\hline Gain & $\begin{array}{c}1034 \\
(64.1 \%)\end{array}$ & $\begin{array}{c}466 \\
(45.1 \%)\end{array}$ & $\begin{array}{c}905 \\
(68.8 \%)\end{array}$ & $\begin{array}{c}263 \\
(24.5 \%)\end{array}$ & $\begin{array}{c}665 \\
(52.8 \%)\end{array}$ \\
\hline
\end{tabular}

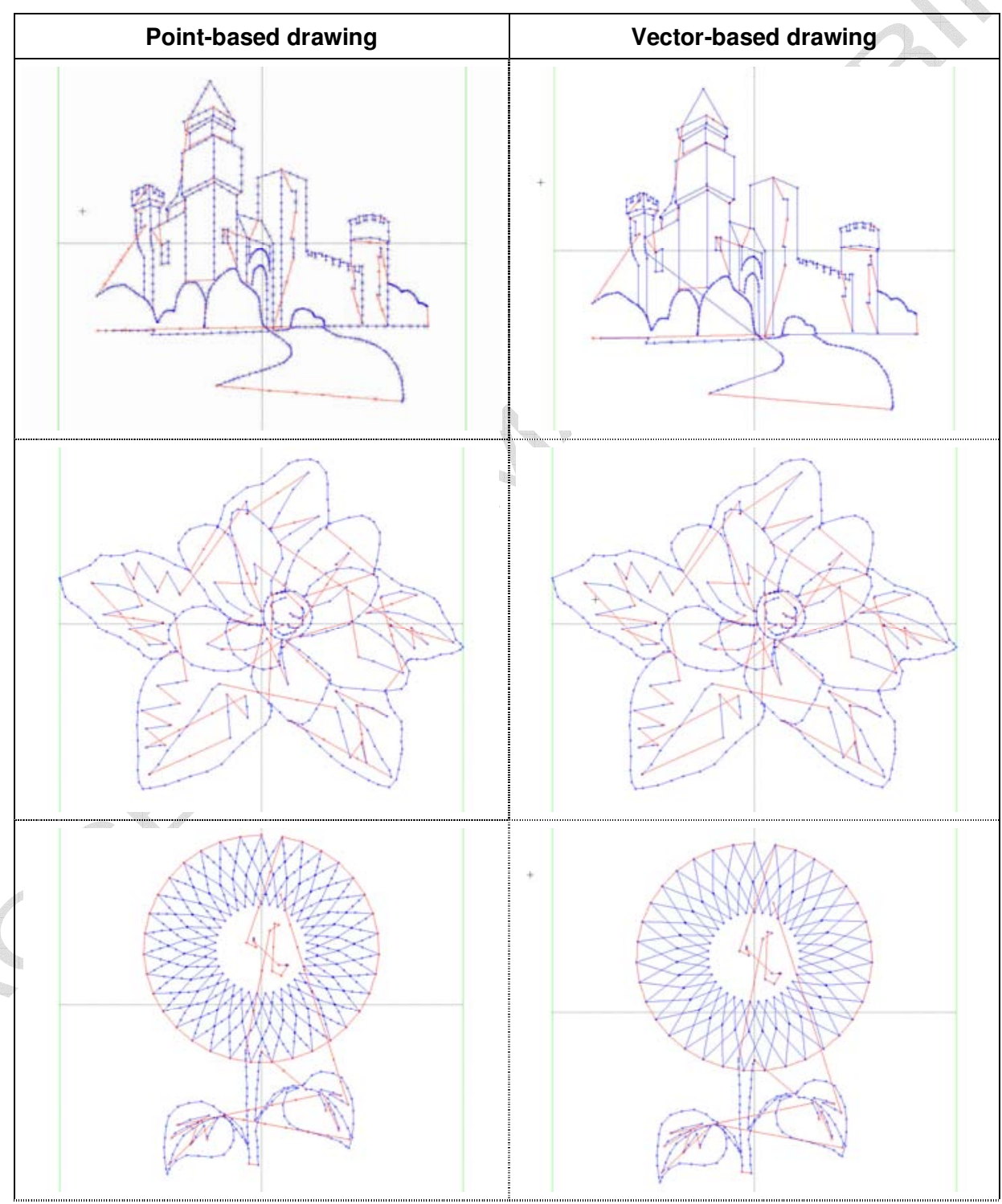




\section{ACCEPTED MANUSCRIPT}

11

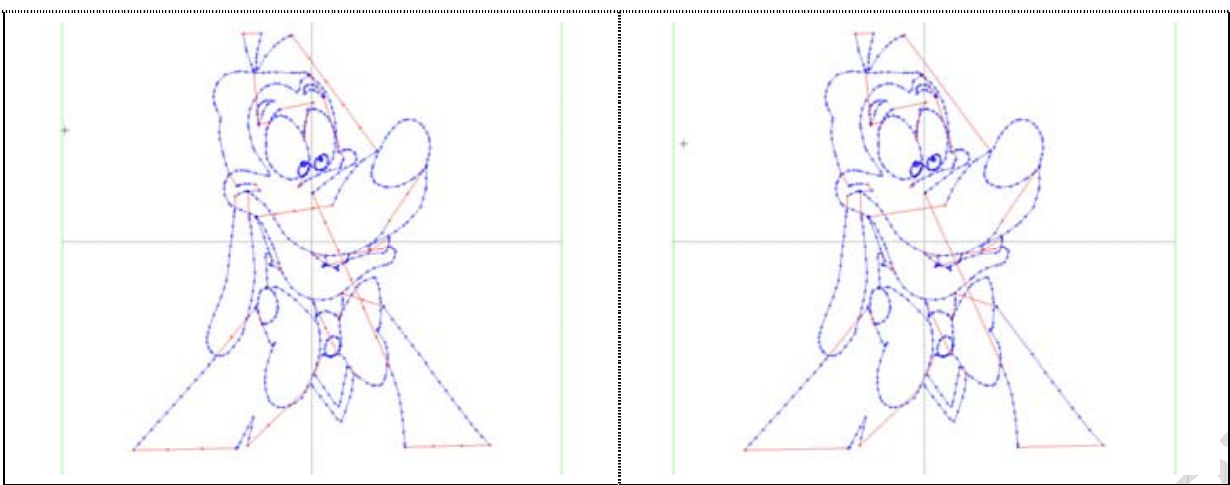

Figure 3: Four different graphics drawing prepared in point-mode and vector-mode.

\subsection{Blanking-Tuning Experiment}

Blanking-command timing (synchronization) is very important to have correct laser-scanned projection as various time delays in the hardware must be taken into consideration. The most critical case is the timing between switching off/on the laser beam and when the mirrors have actually stopped moving.

The main purpose of this experiment is to set up a test to be able to measure the latency of a laser projector and compensate it. We call this procedure 'blanking tuning'. It could be a difficult task as we do not know the exact latency that should be compensated, as explained before. Therefore, a simple test pattern has been proposed to estimate the proper lag/delay times. Projecting parallel lines is a fundamental test for a laser projector, since command timing for blanking is crucial to obtain the correct representation. In order to make the result more general, we tested two patterns of parallel lines as can be seen in Figure 4(a) and Figure 5(a), where blanked lines (hidden lines) could be set vertically or diagonally.

The tested parameter in this experiment is the time that has to be compensated when switching on/off the laser. This delay must be equal to the total laser display latency in order to achieve the accurate synchronization of commands. Therefore, we tuned/adjusted this lag time until two perfectly parallel lines were displayed; the lag time was 20 ticks count (time unit) Figure 4 (e). This time unit depends on the system clock and it differs from one display system to another; therefore, the tuning method presented here is important in order to achieve good laser projection using different laser projectors. In our system, one tick (time unit) equals $23 \mu \mathrm{s}$. If the lag time is not equal to the total laser display latency, we may obtain unexpected results. In the case of switching the laser 
12

off very fast before a scanner delivers the correct voltage to the galvanometer and before the mirror finishes the previous movement (lag < laser display latency), the obtained results are similar to that shown in Figure 4 (c). If the lag time is longer than the total latency of the laser display, then we get the result in Figure 4 (d).

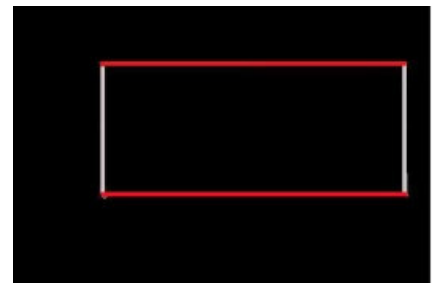

(a) Desired image, white colour represents blanked strokes (hidden strokes).

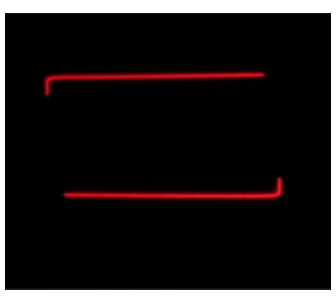

(c) Projected image with lag $=1$

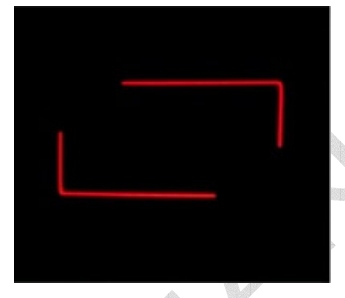

(d) Projected image with

lag $=40$

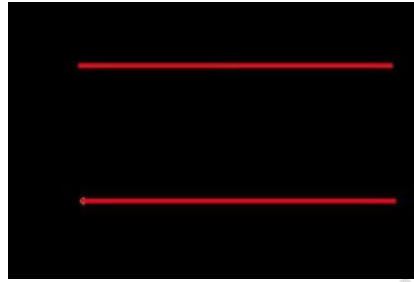

(b) Final projection should be like this image.

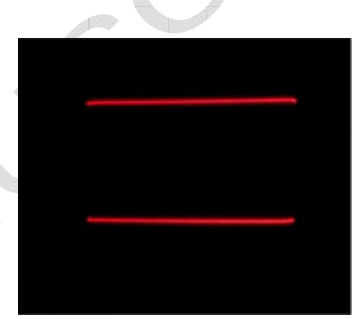

(e) Correct projection achieved

with lag $=20$

Figure 4: Blanking test pattern (1).

The same discussion could be applied to Figure 5 wherein the blanked lines were set diagonally. Different lag time compensation could lead to different results. The most noticeable result is that we could achieve correct projection for the two test patterns at exactly the same lag time (20 time units). Although the test is simple, it can efficiently evaluate the hardware latency/lag in any laser display. 


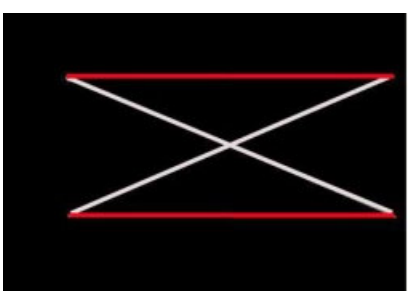

(a) Desired image, white colour represents

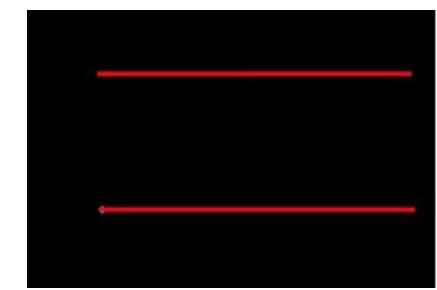

(b) Final projection should be like this image.

blanked strokes.

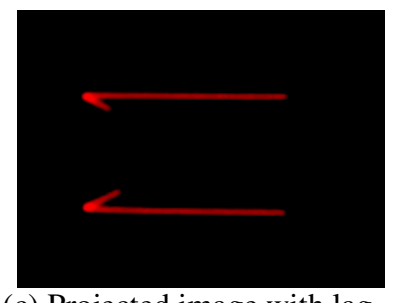

(c) Projected image with lag $=1$

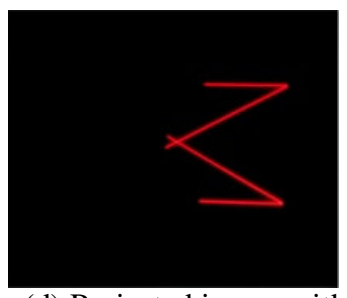

(d) Projected image with

$$
\operatorname{lag}=40
$$

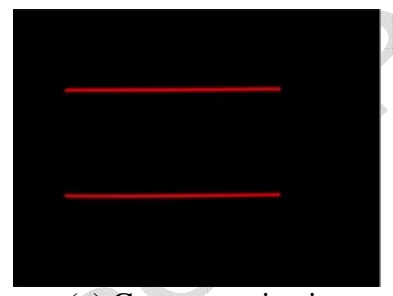

(e) Correct projection

achieved with lag $=20$

Figure 5: Blanking test pattern (2).

\subsection{Optimization of corner dwelling time}

In laser graphics, the laser beam must dwell at corners for about $300 \mathrm{~ms}$ in order to avoid overshooting and ringing. In the point-oriented approach, the dwell time is usually achieved by adding extra points to an image, as described in the previous section. In the proposed vector-oriented approach also, the laser beam must dwell at corners; therefore, we must know how to estimate the proper dwelling time at a corner. In the point-oriented approach, the number of extra points added at the corners and end points are the same, regardless of the angle between the lines that define the corner. Therefore, in order to exactly define the dwelling time for corners and endpoints, an experiment has been carried out to measure the necessary time that should be added.

\subsection{Corner experiment}

A corner test pattern has been proposed to test the necessary dwelling time that has to be considered at the corners to achieve correct laser projection. The test pattern is simply a representation of different angles between $10^{\circ}-180^{\circ}$ with a $10^{\circ}$ increment. The test pattern has been converted to vector data to be displayed by the laser display; see Figure 6. 


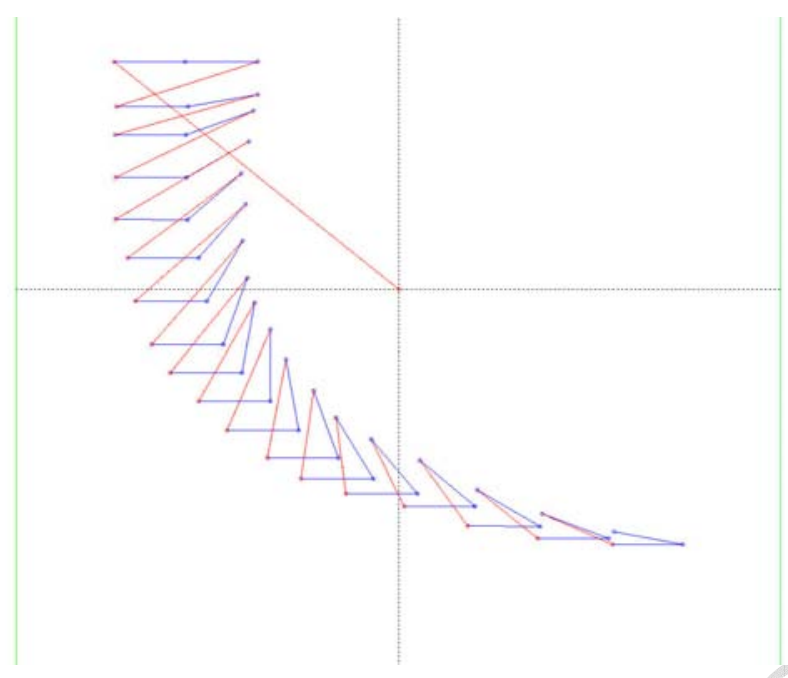

Figure 6: Corner test pattern after converting it to vector data to be displayed on LSD (blue color represents the visible strokes; red color, the blanked strokes).

On the software side, where vector data is processed, we calculate the angle between strokes and add wait time to slow down the laser beam at the corners. The effect of different dwelling times has been examined for the proposed angle pattern. From Figure 7, we can see that when the dwelling time is 0, almost all the corners are projected incorrectly; the corners are rounded, and not sharp. As we increase the dwelling time, the corners gradually become sharp and clear starting from the obtuse angles, where a dwelling time of 2 units is sufficient correctly obtain the angles larger than $160^{\circ}$, meanwhile the angles from $40^{\circ}$ to $160^{\circ}$ still appear rounded. Even though acute angles appear sharp, they are less sharp than they should be. A dwelling time of 6 units was sufficient for corners with angles larger than $120^{\circ}$. By increasing the dwelling time at the corners to more than 12 units, we can notice that all corners appear brighter than earlier until they become very bright dots, as can be seen when the dwelling time equals 40 units. In most software systems, bright dots are clear at corners since these systems set one dwelling time for all the angles; therefore, bright dots can be seen at some corners because of assigning a dwelling time that is larger than that required. Consequently, for correct laser projection, different dwelling times must be set for different angles. Acute angles need more dwelling time than obtuse angles since one mirror has to move to the end point and then switch back. As a result, the dwelling time must be optimized, and by setting one fixed dwelling time for all corners, we are losing some time on the corners that do not necessarily need such time. 
We propose an approach to set the dwelling time according to the corner angle. From our experimental data and the result showed in Figure 7, we could realize that the best approach is to divide the corner angle into nine ranges and accordingly assign the optimum dwelling time. Our choice of setting the dwelling time was practical one based on minimum time for the best result. The right angle was considered the most critical one as it was easy for the viewer to distinguish the quality; therefore, it was assigned more dwelling time. In this manner, we could optimize the dwelling time at the corners more efficiently and saved around $51 \%$ time compared to fixed time or adding fixed points (point-oriented approach) for every corner, as can be seen from Table 2.

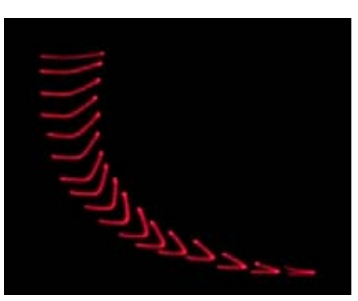

dwelling time $=0$

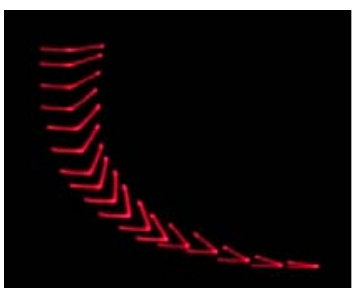

dwelling time $=8$

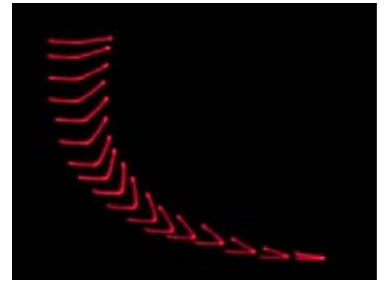

dwelling time $=2$

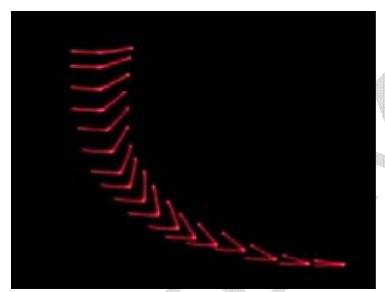

dwelling time $=10$

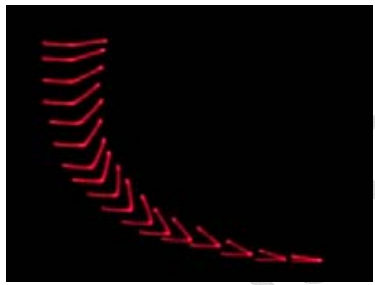

dwelling time $=4$

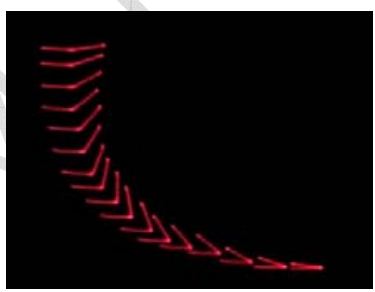

dwelling time $=12$

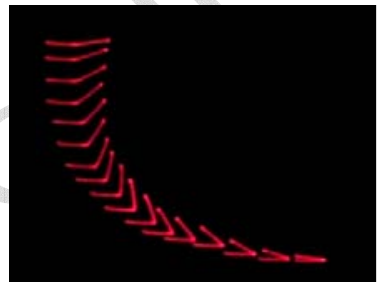

dwelling time $=6$

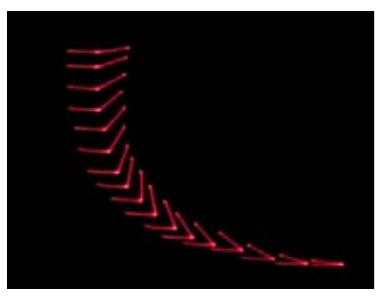

dwelling time $=40$

Figure 7: Laser-scanned corner: test pattern for different dwelling times.

Table 2: The efficiency of dwelling time optimization compared with the point-oriented approach.

\begin{tabular}{|l|c|c|c|c|c|c|c|c|c|c|}
\hline Angle $\left[{ }^{\circ}\right.$ ] & $\begin{array}{c}0- \\
20\end{array}$ & $\begin{array}{c}20- \\
40\end{array}$ & $\begin{array}{c}40- \\
60\end{array}$ & $\begin{array}{c}60- \\
80\end{array}$ & $\begin{array}{c}80- \\
100\end{array}$ & $\begin{array}{c}100- \\
120\end{array}$ & $\begin{array}{c}120- \\
140\end{array}$ & $\begin{array}{c}140- \\
160\end{array}$ & $\begin{array}{c}160- \\
180\end{array}$ & Total \\
\hline $\begin{array}{l}\text { Dwelling time (vector- } \\
\text { oriented) [units } \times 23 \mu \mathrm{s}]\end{array}$ & 10 & 8 & 6 & 4 & 12 & 6 & 4 & 2 & 1 & 53 \\
\hline $\begin{array}{l}\text { Dwelling time (point-oriented) } \\
{[\text { units } \times 23 \mu \mathrm{s} \text { ] }}\end{array}$ & 12 & 12 & 12 & 12 & 12 & 12 & 12 & 12 & 12 & 108 \\
\hline Saved time [units $\times 23 \mu \mathrm{s}$ ] & 2 & 4 & 6 & 8 & 0 & 6 & 8 & 10 & 11 & $\begin{array}{c}55 \\
(50.9 \%)\end{array}$ \\
\hline
\end{tabular}

The optimization efficiency can be increased by lowering the dwelling time for each corner; this may be done on the basis of quality which eventually depends on the designer demands. The data in the table is simply for the 
16

proposed test pattern of a corner angle; therefore, the significance of optimization depends merely on the drawing itself. If the drawing has many acute and obtuse corners, the optimization efficiency may increase; on the other hand, if the drawing has many angles in the $80^{\circ}-100^{\circ}$ range, the efficiency is far lower than expected. Nevertheless, this is a very effective method to optimize the drawing of strokes for laser graphics.

\section{Vector-oriented vs. point-oriented performance}

The vector-oriented approach has many merits over the point-oriented approach: It opens the way for creating, editing and storing object-oriented graphics, as we need only to know an object's feature points to create laser graphics. If each feature point is known, we can easily apply rotation, scaling and other $3 \mathrm{D}$ effects on a specific part. Furthermore, it is not required to add extra points and mid-stroke points which implies a more compact data format. To the best of our knowledge, there is another approach called 'dynamic interpolation' that has been implemented by two European laser companies, Lobo [24] and LaserAnimation [18]. In this approach, midstroke points are generated by the hardware, i.e. dedicated signal-processing chips. We do not know any details on the performance of this approach. Moreover, we do not know if it is pure vector-oriented or point-oriented approach with mid-strokes generated at the hardware level. Therefore, we cannot compare it to the other two approaches.

\subsection{Experimental setup for performance evaluation}

In order to achieve authentic results, commercial software has been used to produce the point-oriented graphics data and we implemented both the vector- and point-oriented approaches to perform all the necessary

measurements on our software. The same drawing was also produced as vector data and both formats were tested on the same laser display.

The test pattern has been specifically designed to include different shapes with various corners of all angles to cover all the possible cases, as shown in Figure 8 (a) and (b). 
17

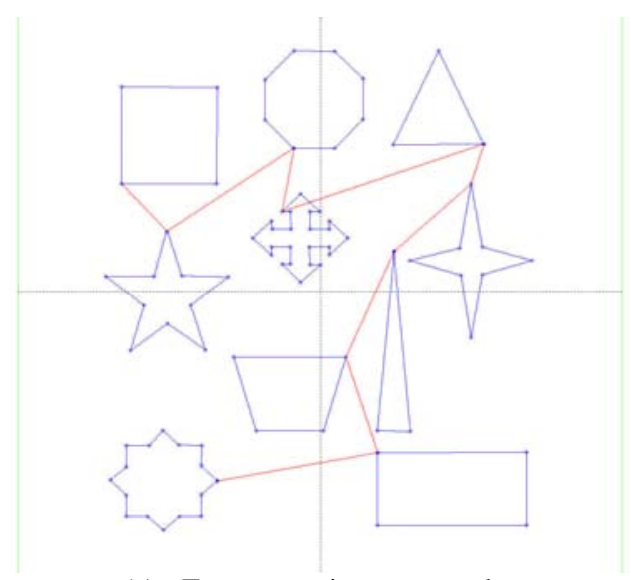

(a) Test pattern in vector mode.

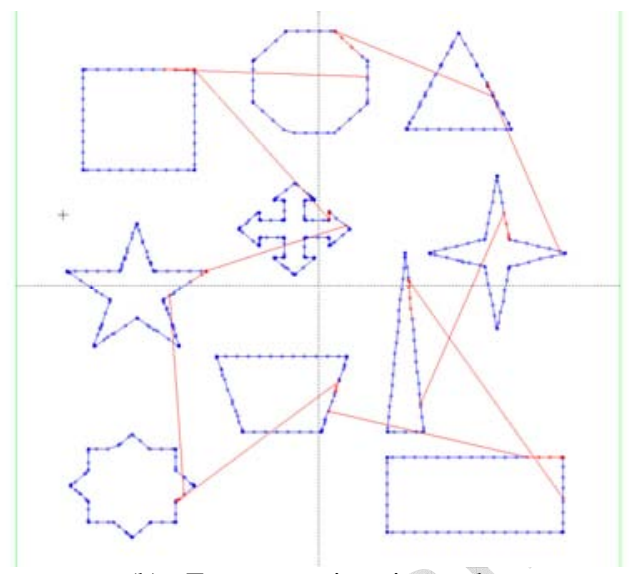

(b) Test pattern in point mode.

Figure 8: test pattern data in vector mode (a) and point mode (b).

These laser graphics drawings have been used to examine the performance of each drawing approach. The refresh rate (frames per second) has been considered as an indicator of the speed performance of each approach. The main target of any laser software is to produce flicker-free laser graphics images. In the point mode, typical frame consists of 400 to 1500 points. In the vector mode, the number of points needed to draw the same drawing is far less than that of the point mode; therefore, comparing the number of points for each frame is infeasible.

Frame rate (FPS) can be calculated from the total time necessary to draw one frame data. As our drawing approach depends on setting the velocity rather than scan rate in point-oriented approach, as explained in the pervious section, the frame rate was calculated for both virtual and real velocities.

The test pattern was tested for different velocities and frame rate and the resulting laser-scanned images were observed, as shown in Figure 9. By changing the velocity, we can draw faster; however, the scanner speed imposes a limitation. A velocity in the range of 20 to $30 \mathrm{~m} / \mathrm{s}$ yielded good images. When the scan speed exceeded $60 \mathrm{~m} / \mathrm{s}$, the projected images started to deform and appeared rather rounded. Nevertheless, the results demonstrate that the proposed vector-oriented approach is capable of drawing laser graphics with the same quality as of that achieved by the point-oriented approach. Moreover, the former approach outperformed the later approach in two aspects: first, we could prevent bright dots from appearing at the corners since we set the exact necessary dwelling time. However, in the point mode, some angles might appear brighter than in the vector mode since the dwelling time is settled for the worst case. The second aspect is that the brightness was constant 
18

for all shapes and for different velocities. Even when the scanners were driven faster, the brightness of each stroke remained unchanged. In the point mode, when the scan speed was increased, the brightness did not remain constant for all shapes; for example, the rectangle and triangle have different brightness in the point mode and the vector mode.

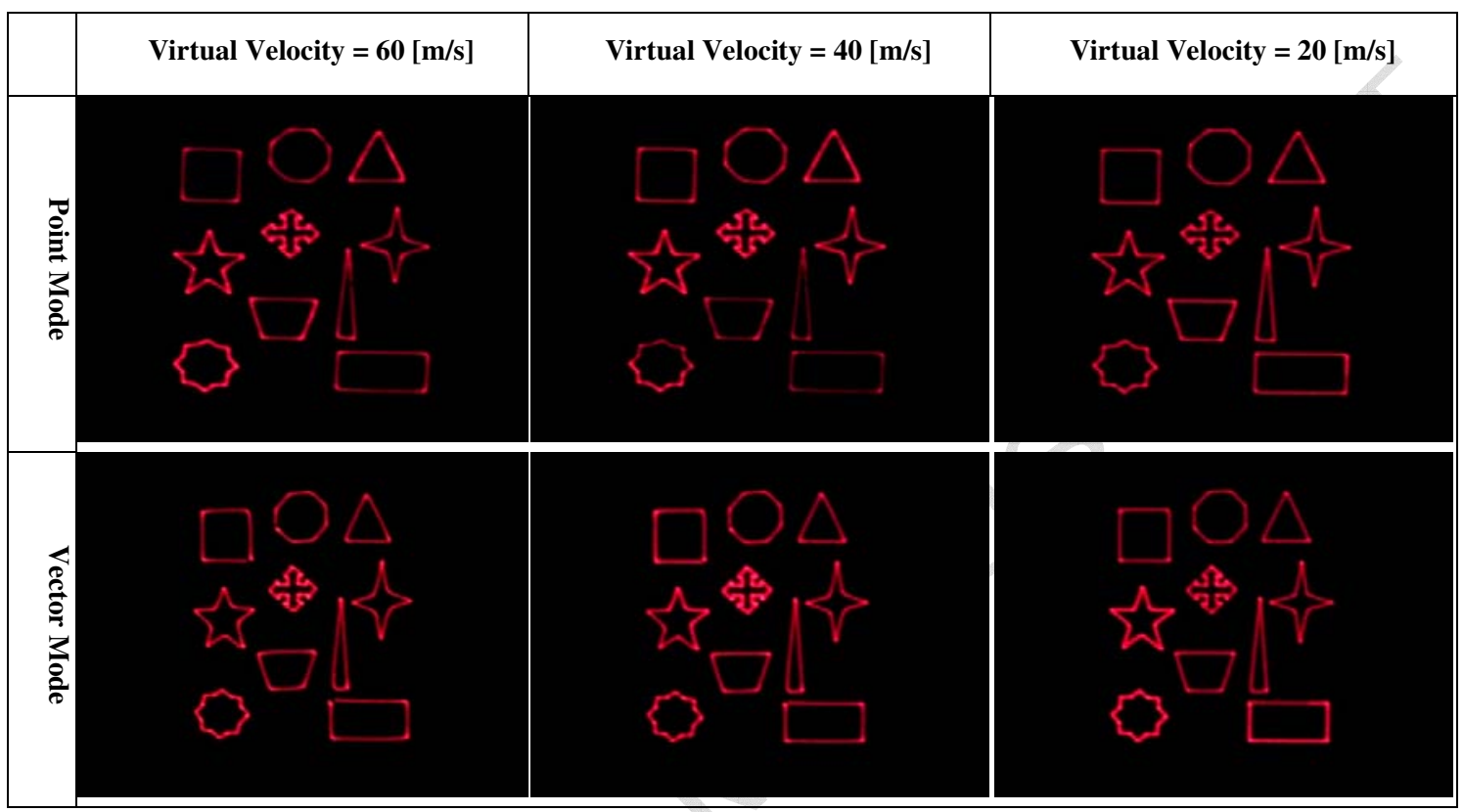

Figure 9. Test patterns that was laser-scanned at different scanning velocities for both vector-oriented mode and point-oriented mode.

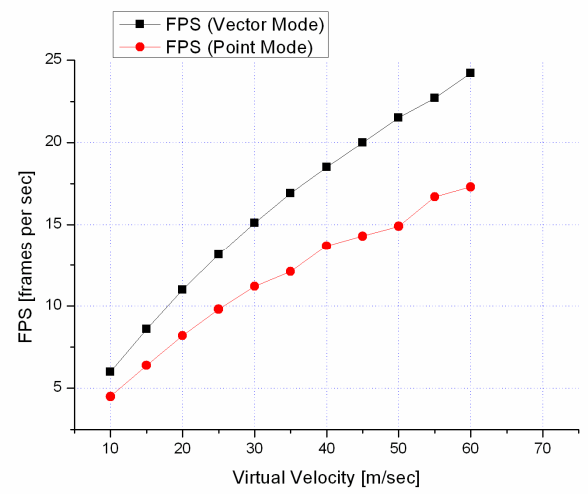

(a)

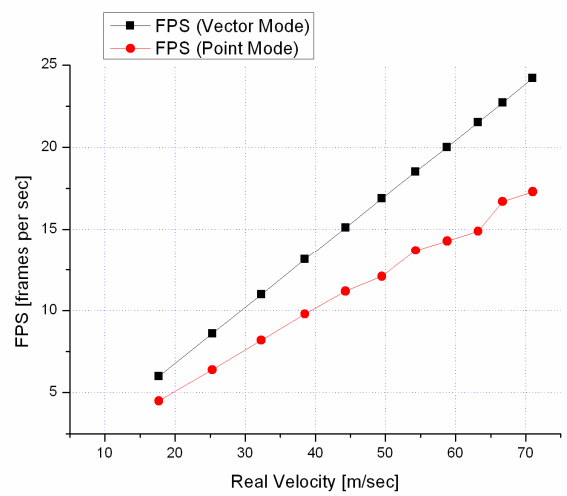

(b)

Figure 10: Performance results in terms of speed (FPS). (a) virtual velocity, (b) real velocity. 
19

The performance of the vector-oriented approach was better than that of the point-oriented approach, as can be seen from Figure 10 (a) and (b). Clearly, there were performance improvements in terms of speed. As a result, we can draw more points in the vector mode which implies that more complex graphics can be drawn with less flickering than in the point mode. The vector mode outperformed the point mode, particularly at high velocities, by around $28.5 \%$, when the velocity was $60[\mathrm{~m} / \mathrm{s}]$. The virtual velocity in Figure 10 (a) is the scanner velocity that can be set by the user. On the contrary, the real velocity in Figure 10 (b) is the one that was calculated from the actual drawing data and the time that was measured by the software. Nonetheless, the performance curve is almost the same for the virtual and real velocities, and only the curve for the virtual one would be sufficient for a user to control the drawing software; however, the real exact result is shown to make our discussion accurate in terms of providing real figures. Additional examples of graphics projected using the presented approach for preparing vector graphics and tuning can be seen in Figure 11.

\section{Conclusion}

In this study, a novel vector-oriented graphic drawing method for LSD was presented. The method considers drawing the strokes directly from the vector data waiving the need to add additional mid-strokes points. The method depends on three new approaches that proposed to achieve perfect and efficient laser projection. Each approach itself is considered to tackle a fundamental problem inherent with laser projection. First, we presented a velocity concept to achieve constant beam intensity for all strokes, the velocity of the laser beam is controlled according to the length of each stroke. This approach is natural, because it uses the motion speed of the laser beam to control the laser drawing. Moreover, the results show that it is very efficient in terms of data reduction; the number of the points to be drawn reduced dramatically (24\% 68\%) compared to the point-oriented method. Furthermore, since there is no need to wait to calculate the total length of one frame data, it is suitable for realtime interactive applications as the beam velocity for each stroke is calculated on fly. Second, the proposed test pattern can decide the hardware compensation time and blanking timing, and can be applied to any LSD. It is simple and sufficient to estimate the latency of any LSD. Third, the optimization approach for corner dwelling

time relies on setting the dwelling time flexibly according to the corner angle. The experimental data and results showed that the dwelling time at the corners could be optimised and time gain was around $50.9 \%$ compared to 
setting fixed time or adding fixed points for every corner. Putting all the described approaches together, a vectororiented drawing method is achieved. It does not require additional points to be inserted; therefore, only the list of end-point vectors is sufficient to project vector graphics.

The performance of the two drawing methods has been examined by testing both approaches on the same pattern image. The vector-oriented approach outperformed the point-oriented approach in terms of speed; this implies that using the former approach, we can draw more vectors and hence display more complex graphics than the latter approach. Nonetheless, the new approach opens the way for creating, editing and storing objectoriented graphics since we need only to know an object's feature points to create laser graphics. If all the feature points of a particular part of an object are known, we can easily apply rotation, scaling and other 3D effects on that specific part. Further more, the elimination of extra points and mid-stroke points implies a more compact data format which can improve the processing and storing performance.

Laser graphics has not yet been explored and researched extensively. Mostly, the industry is developing its own techniques and algorithms to project graphics. We believe that researchers from different fields can collaborate and contribute to hardware and computer graphics in order to achieve effective and efficient laserscanned projection.

\section{Acknowledgment}

This work was partially supported by the Japanese Ministry of Education, Science, Sport and Culture, Grantin-Aid for Exploratory Research 19650014.

\section{References}

[1] SiggraPh2007,

News

Releases, http://www.siggraph.org/s2007/media/releases/release25.html. Retrieved in (Sep. 2008).

[2] N. Glossop, C. Wedlake, J. Moore, T. Peters, Z. Wang, Laser Projection Augmented Reality System for Computer Assisted Surgery. In Proc: Lecture notes in computer science, Vol. 2879, (2003).

[3] Z. Ondogana, O. Pamuka, E. N. Ondogan, A. Ozguney, Improving the appearance of all textile products from clothing to home textile using laser technology. In Proc: Optics \& Laser Technology 37, (2005), 631-637.

[4] Laser projection technologies, Inc. http://www. Iptcorp.com/index.htm. Retrieved in (Sep. 2008).

[5] H. L. Pryor, T. A. Furness, E. Virrre The Virtual Retinal Display: A New Display Technology Using Scanned Laser Light. In Proc: Human Factors and Ergonomics Society, 42nd Annual Meeting, (1998), 1570-1574. 
[6] S. C. McQuaide, E. J. Seibel, J. P. Kelly, B. T. Schowengerdt, T. A. Furness A retinal scanning display system that produces multiple focal planes with a deformable membrane mirror. Displays 24, (2), (2003), 65-72.

[7] G. K. Edgar, H. E. Edgar, P. A.Ward, The effect of viewing a laser-scanned display on colour perception and the visual accommodation response, Displays 29, (2), (2008), 100-105.

[8] J. P. Kelly, S. Turner, H. L. Pryor, E. S. Virrre, E. J. Seibel, T. A. Furness, Vision with a Scanning Laser Display: Comparison of Flicker Sensitivity to a CRT. Displays 2, (5), (2002), 169-175.

[9] S. A. Gonchukov, I. N. Kompanets, Three-dimensional laser display on liquid-crystal medium Laser. Phys. Letter 1, (10), (2004), 511-515.

[10] National institute of advanced industrial science and technology (AIST). Three Dimensional Images in the Air-

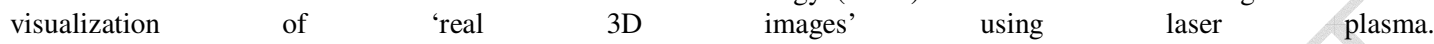
http://www.aist.go.jp/aist_e/latest_research/2006/20060210/20060210.html. Retrieved in (Feb. 2008).

[11] F. J. Menendez, O. Halabi, N. Chiba, Vector-Based Library for Displaying Bezier Curves Using a Laser Projector, In Proc: IWAIT 07 (2007), pp. 121-126.

[12] P. Abderyim, F. J. Menendez, O. Halabi, N. Chiba, Morphing-Based Vectorized Candle Animation for Laser Graphics, In Proc: IWAIT `07 (2007), pp. 127-132.

[13] J.D. Foly, A. VAn Dam, Fundamentals of Interactive Computer Graphics, Addison-Wesley, 1984.

[14] J. COLLINC, D. TuCKER, Laser Graphics and Animation, BYTE 9, 10 (1984), 177-184.

[15] T. Strothotte, S. Schlechtweg, Non-photorealistic computer graphics, Morgan Kaufann, 2002.

[16] M. Iri, K. murota, S. Matsui, An approximate solution for the problem of optimizing the plotter pen movement, System Modeling and Optimization. In Proc: Lecture Notes in Control and Information Sciences, Vol. 38, (1983), 572-580.

[17] PAngolin, lasershow software, http://www.pangolin.com/. Retrieved in (Jan. 2008).

[18] LaSERAnimation (Sollinger), http: //www. laseranimation. com/. Retrieved in (Sep. 2008).

[19] Phoenix, the professional showcontroller, http://www.phoenix-lasersoftware.de/. Retrieved in (Sep. 2008).

[20] Medialas, Mamba, lasershow software, http://www.medialas.de/showlaser/software_e.htm. Retrieved in (Sep. 2008).

[21] P. Murphy, B. Benner, Computer Graphics for Scanned Laser Displays, In Proc: SMPTE Technical Conference, (Nov. 1992), 134-78.

[22] GSILumONICS, Optical Scanners and Controllers, http://www.gsig.com/, Retrieved in (Sep. 2008).

[23] Coherent, Coherent Lasers, http://www. coherent. com/, retrieved in (Sep. 2008).

[24] LOBO electronics, http://www. lobo. de/, Retrieved in (Sep. 2008). 


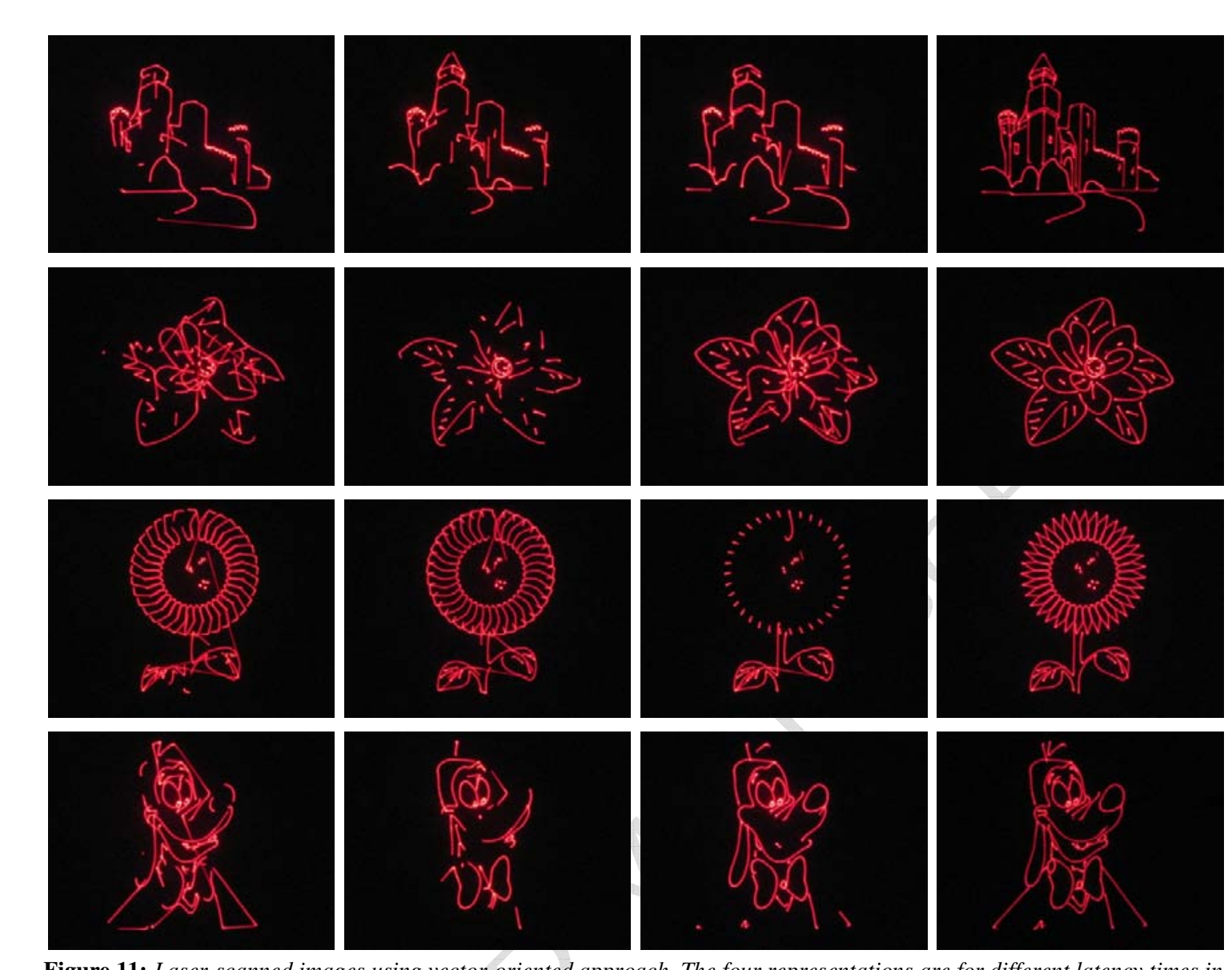

